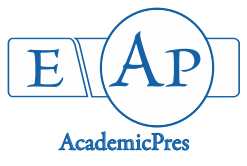

Falodun EJ and Edafe VE (2020)

Notulae Scientia Biologicae 12(3):719-728

DOI: $10.15835 / \mathrm{nsb} 12310754$

Research Article

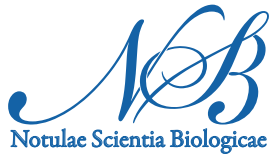

\title{
Productivity, nutrient concentration, uptake and quality of Amaranthus (Amaranthus cruentus L.) as influenced by cutting heights and fertilizer type
}

\author{
Ehizogie J. FALODUN*, Vivian E. EDAFE \\ University of Benin, Faculty of Agriculture, Department of Crop Science, \\ Nigeria; ehizogie.falodun@uniben.edu (*correspondingauthor)
}

\begin{abstract}
Amaranthus cruentus $\mathrm{L}$. is a leafy vegetable that is cultivated mainly for its leaves and grains. Due to high demand for this crop in Nigeria, the need to boost its productivity becomes necessary. Field and laboratory studies were conducted during in two successive cropping seasons at the experimental farm and laboratory unit of the Department of Crop Science, Faculty of Agriculture, University of Benin, Benin City. Three cutting heights C1: $(10 \mathrm{~cm}), \mathrm{C} 2:(15 \mathrm{~cm}), \mathrm{C} 3:(20 \mathrm{~cm})$ and a control C0: (no cutting) and four rates of fertilizer

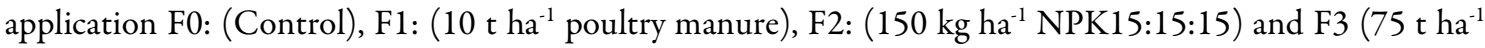
NPK 15:15:15 $+5 \mathrm{t} \mathrm{ha}^{-1}$ poultry manure) constituted the treatments. The treatments were in three replicate laid out in a $(4 \times 4)$ factorial arrangement in a randomized complete block design. Data collected were plant height $(\mathrm{cm})$, leaf area $\left(\mathrm{cm}^{2}\right)$, stem diameter $(\mathrm{cm})$ and number of branches. Fresh and dry herbage yield were taken at harvest. Growth, yield, and quality of Amarathus cruentus were significantly influenced by cutting heights and fertilizer application. In both years, number of branches (3.50 and 6.01) were lowest at C0 compared with other treatments. Herbage fresh and dry yield $\left(49.10 \mathrm{t} \mathrm{ha}^{-1}\right.$ and $\left.18.20 \mathrm{t} \mathrm{ha}^{-1}\right)$ were highest at C3 and was consistent in both years. Similarly, the concentration of $\mathrm{K}, \mathrm{Ca}$, and $\mathrm{Mg}$ in plant tissue and the uptake of nutrients $(\mathrm{N}, \mathrm{P}, \mathrm{K}, \mathrm{Ca}, \mathrm{Mg}$, and $\mathrm{Na}$ ) increase at $\mathrm{C} 3$ compared with other treatments. F3 significantly $(\mathrm{p}<0.05)$ produced highest dry herbage yield $\left(13.08 \mathrm{t} \mathrm{ha}^{-1}\right.$ and $\left.25.15 \mathrm{t} \mathrm{ha}^{-1}\right)$ in both years. Crude protein, fibre and fat content of the shoots were highest at cutting height of $\mathrm{C} 3$ and at $\mathrm{F} 3$ fertilizer rate. Therefore, for good yield and better quality of Amaranthus cruentus $\mathrm{L}$, a combination of $\left(75 \mathrm{t} \mathrm{ha}^{-1} \mathrm{NPK} 15: 15: 15+5 \mathrm{tha}^{-1}\right.$ poultry manure) at cutting height of C3: (20 cm above soil level) is recommended for Amaranthus cruentus $\mathrm{L}$ production in the study area.
\end{abstract}

Keywords: herbage yield; nutrient uptake; plant tissue; vegetables

\section{Introduction}

Amaranthus cruentus L. commonly known as Amaranth belongs to the family Amaranthaceae. It is a dual-purpose crop, grown both for its leaves and grains. A. cruentus is a vegetable of high dietary value produced and consumed in most parts of Nigeria. Nutritionally, the leaves are rich in protein, vitamin A and potassium (Tindall, 1986; Akanbi and Togun, 2002). The leaves are being processed into many food items, supplements, 
and additives (NIHORT, 1986; Ojo and Olufolaji, 1987). Despite the popularity of this vegetable in traditional cropping system, the production and nutritional values of these vegetables are limited due to poor agronomic practices and low fertility of native soils in most parts of Nigeria (Law-Ogbomo et al., 2012). Efforts to develop and use fertilizer that will boost agricultural production and at the same time be friendly to the environment need be encouraged. The use of animal residues such as poultry manure for the growth and yield of vegetable and other crops had been advocated because of their low cost and availability (Moyin-Jesu, 2002). A continual dependence on chemical fertilizers may be accompanied by a fall in organic matter content, increased soil acidity, degradation of soil physical properties and increased rate of erosion due to instability of soil aggregates (Adeoluwa and Akinyemi, 2014). One of the ways to maintain or improve the soil fertility is by maintaining its organic matter. Vegetable farmers mostly apply poultry manure in combination with inorganic nitrogen-based fertilizers such as urea and NPK often because poultry manure alone is believed to dissolve slowly and may not meet up the yield of vegetables. The combine use of organic or inorganic fertilizers can give the desired higher sustainable crop yields than sole use of organic or inorganic fertilizer (Adeoye et al., 2008; Akanbi et al., 2010; Gundlade et al., 2011). Falodun et al. (2013) reported that complimentary use of organic fertilizers can give the desired higher sustainable crop yields than sole use of inorganic fertilizer. Amaranth, like other leaf vegetables can be harvested either by uprooting (NIHORT, 1986) or repeated cutting back of the shoots until when inflorescences appear on the main trunks. Repeated cutting when done at adequate height and interval was observed to produce higher shoot yield of Corchorus sp. per unit of land (NIHORT, 1986). Stem cuttings at several intervals above the ground permit the lateral shoots to grow so that harvesting can be done a greater number of times. This is more economical and produces higher returns when compared with the situation in which the plants were uprooted once. Spreading of harvest over time also stabilizes produce, market price and hence higher returns. Mnzava and Masam (1985) reported that leaf yield depends on the portion of leaves and buds left behind for re-growth. They also found $20 \mathrm{~cm}$ initial cutting height as most suitable for $A$. cruentus compared to 3,10 and $15 \mathrm{~cm}$. These results therefore clearly show that less destructive cutting heights were beneficial for amaranth species probably due to more leaf and buds remaining on the stem consequently resulting in a high level of auxin for re-growth. The objective of this study is to determine the effect of cutting height and fertilizer type on productivity, nutrient concentration, uptake, and quality of Amaranthus cruentus (L).

\section{Materials and Methods}

\section{Experimental site and soil properties}

The field experiment was conducted for two consecutive crop cycles (2017-2019) at the Experimental Farm of the Department of Crop Science, Faculty of Agriculture, University of Benin, Benin City, Nigeria. The location lies between latitude $6^{\circ} 14^{\prime} \mathrm{N}$ and $7^{\circ} 34^{\prime} \mathrm{N}$ and longitude $5^{\circ} 40^{\prime} \mathrm{E}$ and $6^{\circ} 43^{\prime} \mathrm{E}$ on elevation of 162 $\mathrm{m}$ above sea level. The monthly rainfall distribution pattern for the area is bimodal with peaks in June and September. Annual rainfall ranges from 1200 to $1450 \mathrm{~mm}$ spanning over eight months (March to October) with a dry spell in August. The dry season is from November to March. Prior to laying out of the experiment, composite soil samples collected at a depth of $0-5 \mathrm{~cm}$ from the top soil were air-dried, sieved, and packaged for routine soil physicochemical analysis. Soil $\mathrm{pH}$ was determined using a $\mathrm{pH}$ meter. Organic carbon was determined by wet oxidation method (Walkley and Black, 1962) as modified by Jackson (1969). Total nitrogen was obtained by macro Kjeldahl method as modified by Jackson (1969). Available P was extracted by Bray I method (Bray and Kurtz, 1945) and P was estimated by the blue colour method of Murphy and Riley (1962). Exchangeable $\mathrm{K}$ and $\mathrm{Na}$ were determined using flame photometer, while $\mathrm{Ca}$ and $\mathrm{Mg}$ were determined using the Atomic Absorption Spectrophotometer. The results of the soil analysis and swine manure are presented in collected at a depth of $0-15 \mathrm{~cm}$ from the top soil were air - dried, sieved and subjected to routine soil analysis using standard laboratory procedures (Table1). 
Falodun EJ and Edafe VE (2020). Not Sci Biol 12(3):719-728

Table 1. Physico-chemical composition of soil from experimental site pre planting

\begin{tabular}{|c|c|}
\hline $\mathrm{pH}\left(\mathrm{H}_{2} \mathrm{O}\right)$ & 5.65 \\
\hline Phosphorus $\left(\mathrm{mg} \mathrm{kg}^{-1}\right)$ & 6.02 \\
\hline Total Nitrogen $(\%)$ & 0.03 \\
\hline Exchangeable bases $\left(\mathrm{cmol} \mathrm{kg}^{-1}\right)$ & 0.65 \\
\hline Calcium & 0.20 \\
\hline Magnesium & 0.12 \\
\hline Potassium & 0.13 \\
\hline Sodium & 17.12 \\
\hline Organic matter $(\%)$ & 89.20 \\
\hline Sand $(\%)$ & 5.30 \\
\hline Silt (\%) & 5.50 \\
\hline Clay $(\%)$ & Sandy loam \\
\hline Textural class & \\
\hline
\end{tabular}

\section{Source of planting material}

$A$. cruentus seeds and poultry manure used for the experiment were obtained from the Department of Crop Science Farm and Animal Science Farm Unit of University of Benin respectively, while NPK 15:15:15 fertilizer was obtained from Agricultural Development Programme (ADP), Edo State, Nigeria.

\section{Experimental design}

Raised beds $1 \times 1 \mathrm{~m}$ with a boundary of $0.5 \mathrm{~m}$ were manually prepared and laid out in a $4 \times 4$ factorial arrangement fitted into a randomised complete block design (RCBD) with 16 treatments replicated 3 times. The treatments were based on four cutting heights C0: (no cutting), C1: $(10 \mathrm{~cm}), \mathrm{C} 2:(15 \mathrm{~cm}), \mathrm{C} 3:(20 \mathrm{~cm})$ and four rates of fertilizer application F0: (Control), F1: (10 t ha-1 poultry manure), F2: (150 kg ha-1 NPK15:15:15) and F3: (75 kg ha-1 NPK 15:15:15 + $5 \mathrm{t} \mathrm{ha}^{-1}$ poultry manure). The poultry manure used for the experiment was incorporated into their respective plots, depending on the treatment, and left for two weeks before transplanting of seedlings for equilibration. Seeds of $A$. cruentus were sown through broadcasting in the nursery and transplanted to the field when seedlings were three weeks after sowing (WAS) at one seedling per stand at a spacing of $20 \times 20 \mathrm{~cm}$ giving a plant population of 250,000 plants $\mathrm{ha}^{-1}$. Plots were mulched to conserve soil moisture; weeding was done first at two weeks after transplanting and subsequently weeding was done as soon as weeds emerged.

\section{Sampling and measurement}

Data collection started four weeks after transplanting (WAT) and continued fortnightly thereafter. Four plants were randomly selected from each plot and tagged for the purpose of collecting data for plant height $(\mathrm{cm})$, number of leaves, number of branches, leaf area $(\mathrm{cm})$ and stem diameter $(\mathrm{cm})$. Harvesting of plants was by cutting the plants at 10,15 and $20 \mathrm{~cm}$ above soil surface as per treatment. This was repeated fortnightly until when inflorescence appeared at (10 WAT) on the non-cut plants or on the side shoots of the cut plants. A total of four cuttings were made. The cumulative herbage fresh yield was determined at the end of the last harvest for all the treatment except for the non-cut plant treatment in which shoot harvesting was done once at 10 weeks after sowing and used to estimate its herbage fresh yield in $\left(\mathrm{t} \mathrm{ha}^{-1}\right)$ based on the formula below

$$
\text { Yield (tonnes per hectare) }=\frac{\text { Plot yield (tonnes) } x 10000}{\text { Plot size (metre sq.) }}
$$

For dry herbage yield, fresh shoot samples were chopped and packed inside well labelled envelopes and dried in oven at $70{ }^{\circ} \mathrm{C}$ to attain a constant weight. This was done periodically (for the cut plants) and total dry herbage yield (for non - cut plants). The dried samples were there after weighed using a sensitive electronic 
balance and the weight recorded. The cumulative dry herbage yields were obtained by adding together individual value obtained at each harvest and estimated in $\left(\mathrm{tha}^{-1}\right)$.

\section{Determination of tissue nutrient concentration, uptake and proximate analysis of Amaranthus cruentus} leaves

At 70 DAT (at harvest leaves were harvested, cleaned of soil particles with distilled water and air dried and weighed with a sensitive balance. The leaves were chopped into small bits and packed inside well labelled envelopes and oven dried in a forced-Air Sanyo Gallen kamp moisture extraction oven at $70{ }^{\circ} \mathrm{C}$ to a constant weight (ISTA, 1993). The dried samples were there after weighed using a sensitive electronic balance and the weight recorded. These were milled to pass through $0.2 \mathrm{~mm}$ sieve using Thomas Wiley Hammer Mill in preparation for laboratory analysis. Proximate composition was analysed using the AOAC (1990) method. The components of proximate composition are crude protein $(\mathrm{CP})$, crude fiber $(\mathrm{CF})$, and ether extract $(\mathrm{EE})$, ash, moisture, and carbohydrate content. Calcium $(\mathrm{Ca})$, Sodium $(\mathrm{Na})$ and Magnesium $(\mathrm{Mg})$ along with $\mathrm{N}, \mathrm{P}$ and $\mathrm{K}$ were determined following standard laboratory procedures. The analytical procedures for the nutrients were as described by AOAC (2000). Nitrogen, P, K, Ca, Na and Mg uptakes were calculated using the formula:

Nutrient uptake $=$ oven dry weight of tissue $\mathrm{x}$ nutrient content in plant tissue $(\%)$.

\section{Statistical analysis}

Data collected were subjected to analysis of variance (ANOVA), using SAS (Statistical Analysis Software) and least significance difference (LSD) test at $5 \%$ level of probability was used to compare the significant treatment mean.

\section{Results}

The chemical characteristics of the soil used for the experiment showed that the soil was strongly acidic and low in the essential soil nutrients (Table1). Significant $\mathrm{P} \leq 0.05$ differences were observed in some of the agronomic parameters measured as influenced by cutting heights and fertilizer application. In 2017/18, irrespective of the cutting height, plants that were cut produced significantly similar and higher number of leaves and branches compared to the non-cut plants. The number of leaves increased from (36.34 to 67.41) for the cut plants and number of branches (3.50) was lowest with the non-cut plants and increased to (7.24) when plants were cut at $\mathrm{C} 3$ while the leaf area $\left(32.29 \mathrm{~cm}^{2}\right)$ was highest at $\mathrm{C} 0$ (the non - cut plants) and increased significantly above plants that were cut. In 2018/19, the number of leaves (80.53) was highest at C2 (plants cut at $15 \mathrm{~cm}$ above soil level) while the stem diameter $(0.45)$ was least with plants that were not cut (Table 2). Similarly, plants that received fertilizers produced significantly higher number of leaves and stem diameter compared with the non-fertilize plants in both years. In 2017/18 season there were significant differences in all the yield characters measured, $C 3$ (plants cut at $20 \mathrm{~cm}$ above soil surface) significantly $\mathrm{P} \leq 0.05$ produced higher fresh herbage weight per plant $(20.42 \mathrm{~g})$, fresh and dry herbage yield $\left(49.10\right.$ and $\left.18.20 \mathrm{tha}^{-1}\right)$ above other cutting heights (Table 2). In 2018/2019, irrespective of cutting height, plants cut at $10 \mathrm{~cm}(\mathrm{C} 1), 15 \mathrm{~cm}$ (C2) and (C3) $20 \mathrm{~cm}$ were similar and enhanced fresh herbage weight per plant $(32.54,26.12$ and $20.27 \mathrm{~g})$ and fresh herbage yield $\left(67.10,57.41\right.$ and $\left.48.40 \mathrm{t} \mathrm{ha}^{-1}\right)$ compared with the non-cut plants $(\mathrm{C} 0)$ which significantly produced the lowest values of $15.39 \mathrm{~g}$ and $37.00 \mathrm{t} \mathrm{ha}^{-1}$ respectively for these parameters. Plants cut at $20 \mathrm{~cm}$ above soil level (C3), consistently produced the highest fresh and dry herbage yield in both years. Amaranths plants performed better with application of fertilizer compared to non- fertilized plants, fertilizer application had a significant $(\mathrm{P} \leq 0.05)$ effect on the yield attributes. The dry herbage yield increased from 9.21 to 15.99 and 18.92 and 25.15 t ha ${ }^{-1}$ when (F2) and (F3) fertilizer rates were used respectively. In 2017/18, application of F2 and F3 significantly $(\mathrm{p}<0.05)$ produced similar highest dry herbage yield of $\left(15.99\right.$ and $\left.13.08 \mathrm{t} \mathrm{ha}^{-1}\right)$ respectively. However, F3 consistently produced the highest dry herbage yield (13.08 and 25.15) in both years (Table 2). 
Cutting height increased nutrient concentration of $\mathrm{K}$ from ( 0.27 to $\left.0.44 \mathrm{~g} \mathrm{~g}^{-1}\right), \mathrm{Mg}\left(0.37\right.$ to $\left.0.63 \mathrm{~g} \mathrm{~g}^{-1}\right)$ and $\mathrm{Ca}$ ( 2.10 and $3.32 \mathrm{~g} \mathrm{~g}^{-1}$ ) while $\mathrm{N}, \mathrm{K}$ and $\mathrm{Ca}$ concentrations were increased with fertilizer applied as sole inorganic (F2) or combination of organic and inorganic fertilizer (F3) (Table 3). Nutrient uptake increased with increase in cutting height. Uptakes ( $\left.\mathrm{Kg} \mathrm{ha}^{-1}\right)$ of $\mathrm{N}$ (1.67), $\mathrm{P}(41.01), \mathrm{K}(24.08), \mathrm{Ca}(180.58)$ and $\mathrm{Na}(0.35)$ were highest at (C3) plants cut at $20 \mathrm{~cm}$ above soil level (Table 4). Similarly, plants that received fertilizers increased nutrient uptake above the non - fertilized plants. The moisture content on dry matter basis (3.50\%) was highest at cutting height of $15 \mathrm{~cm}$ above ground level while the ash (5.70\%) and percentage crude protein content (5.03\%), fat (7.92\%) and fibre (6.22\%) increased with increase in cutting height and was highest at (C3) cutting at $20 \mathrm{~cm}$ above ground. Moisture contents (3.00 and 3.00\%) were similar and higher in F0 (no fertilizer application) and F1 (10 t ha ${ }^{-1}$ sole poultry manure application) (Table 5). The percentage ash and crude protein content (5.40 and 4.26\%) were highest at F2 and F3 respectively and lowest at F0 (4.12 and 1.84\%). Carbohydrate content decreased with increase in cutting height and fertilizer application and was highest at $\mathrm{C} 0(83.93 \%)$ and F0 (81.46\%). The use of F3 (75 kg ha ${ }^{-1} \mathrm{NPK} 15: 15: 15+5 \mathrm{t} \mathrm{ha}^{-1}$ poultry manure), increased the crude fat (6.70\%) and fibre content (4.62\%) of amaranths leaves above other treatments (Table 6).

Table 2. Effect of cutting height and fertilizer application on some vegetative characters of Amaranthus cruentus L.

\begin{tabular}{|c|c|c|c|c|c|c|c|c|c|c|}
\hline \multirow[t]{2}{*}{ Treatment } & \multicolumn{5}{|c|}{ 2017/2018 Cropping season } & \multicolumn{5}{|c|}{ 2018/2019 Cropping season } \\
\hline & $\begin{array}{l}\text { Plant } \\
\text { height } \\
(\mathrm{cm})\end{array}$ & $\begin{array}{l}\text { Number } \\
\text { of leaves }\end{array}$ & $\begin{array}{c}\text { Number } \\
\text { of } \\
\text { branches }\end{array}$ & $\begin{array}{l}\text { Leaf } \\
\text { area } \\
\left(\mathrm{cm}^{3}\right)\end{array}$ & $\begin{array}{c}\text { Stem } \\
\text { diameter } \\
(\mathrm{cm})\end{array}$ & $\begin{array}{l}\text { Plant } \\
\text { height } \\
(\mathrm{cm})\end{array}$ & $\begin{array}{l}\text { Number } \\
\text { of leaves }\end{array}$ & $\begin{array}{c}\text { Number } \\
\text { of } \\
\text { branches }\end{array}$ & $\begin{array}{c}\text { Leaf } \\
\text { area } \\
\left(\mathrm{cm}^{3}\right)\end{array}$ & $\begin{array}{c}\text { Stem } \\
\text { diamete } \\
(\mathrm{cm})\end{array}$ \\
\hline \multicolumn{11}{|l|}{$\begin{array}{l}\text { Cutting height } \\
(\mathrm{cm})\end{array}$} \\
\hline $\mathrm{C} 0$ & $45.79 \mathrm{a}$ & $36.34 \mathrm{~b}$ & $3.50 \mathrm{~b}$ & $32.29 \mathrm{a}$ & $2.52 \mathrm{a}$ & $21.61 \mathrm{~b}$ & $48.05 \mathrm{c}$ & $6.01 \mathrm{~b}$ & $22.20 \mathrm{a}$ & $0.45 b$ \\
\hline $\mathrm{C} 1$ & $38.99 a$ & $42.62 \mathrm{a}$ & $5.65 \mathrm{a}$ & $18.44 \mathrm{~b}$ & $2.27 \mathrm{ab}$ & $36.19 \mathrm{a}$ & $45.15 c$ & $10.15 \mathrm{a}$ & $17.27 \mathrm{a}$ & $0.99 \mathrm{a}$ \\
\hline $\mathrm{C} 2$ & $37.26 a$ & $48.66 a$ & $6.90 \mathrm{a}$ & $16.61 \mathrm{~b}$ & $2.05 \mathrm{a}$ & $44.28 \mathrm{a}$ & $80.53 a$ & $13.27 \mathrm{a}$ & $25.03 \mathrm{a}$ & $1.30 \mathrm{a}$ \\
\hline C 3 & $39.63 a$ & $67.41 \mathrm{a}$ & $7.24 a$ & $16.83 \mathrm{~b}$ & $2.31 \mathrm{ab}$ & $45.17 \mathrm{a}$ & $59.44 \mathrm{~b}$ & $10.83 a$ & $28.40 \mathrm{a}$ & $1.21 \mathrm{a}$ \\
\hline Significance & Ns & ${ }^{*}$ & ${ }^{*}$ & ${ }^{*}$ & ${ }^{*}$ & ${ }^{*}$ & ${ }^{*}$ & ${ }^{*}$ & Ns & ${ }^{*}$ \\
\hline $\operatorname{Lsd}_{(0.05)}$ & 13.22 & 7.79 & 2.08 & 6.68 & 0.31 & 10.20 & 4.67 & 3.31 & 31.06 & 0.35 \\
\hline \multicolumn{11}{|l|}{ Fertilizer rates } \\
\hline F 0 & $42.01 \mathrm{a}$ & $4327 b$ & $7.81 \mathrm{a}$ & $18.68 \mathrm{a}$ & $2.20 \mathrm{a}$ & $34.31 \mathrm{a}$ & $4752 b$ & $9.17 \mathrm{a}$ & $47.22 \mathrm{a}$ & $0.79 \mathrm{~b}$ \\
\hline F 1 & $42.20 \mathrm{a}$ & $57.42 \mathrm{a}$ & $8.30 \mathrm{a}$ & $23.81 \mathrm{a}$ & $2.25 \mathrm{a}$ & $40.02 a$ & $60.21 \mathrm{a}$ & $9.90 \mathrm{a}$ & $57.23 a$ & $1.17 \mathrm{a}$ \\
\hline F 2 & $32.58 \mathrm{a}$ & $56.18 \mathrm{a}$ & $8.54 a$ & $19.08 \mathrm{a}$ & $2.44 a$ & $36.11 \mathrm{a}$ & $67.14 a$ & $11.65 a$ & $50.43 a$ & $1.11 \mathrm{a}$ \\
\hline F 3 & $34.99 a$ & $57.50 \mathrm{a}$ & $8.60 \mathrm{a}$ & $17.63 a$ & $2.25 \mathrm{a}$ & $38.12 \mathrm{a}$ & $66.18 \mathrm{a}$ & $12.21 \mathrm{a}$ & $52.15 \mathrm{a}$ & $1.15 \mathrm{a}$ \\
\hline Significance & Ns & ${ }^{*}$ & $\mathrm{Ns}$ & $\mathrm{Ns}$ & Ns & ns & $*$ & ns & ns & ${ }^{*}$ \\
\hline $\operatorname{Lsd}_{(0.05)}$ & 13.22 & 7.79 & 2.08 & 6.58 & 0.31 & 10.20 & 4.67 & 3.31 & 31.06 & 0.35 \\
\hline \multicolumn{11}{|l|}{ Interaction } \\
\hline $\mathrm{C}^{*} \mathrm{~F}$ & Ns & Ns & Ns & Ns & Ns & ns & Ns & $\mathrm{ns}$ & $\mathrm{ns}$ & ns \\
\hline
\end{tabular}

Means followed by the same alphabets along rows are not significantly different at $5 \%$ level of probability C0: No cutting, C1: $(10 \mathrm{~cm}), \mathrm{C} 2:(15 \mathrm{~cm}), \mathrm{C} 3:(20 \mathrm{~cm})$ above soil level.

F0: Control, F1: $10 \mathrm{t} \mathrm{ha}^{-1}$ poultry manure, F2: $150 \mathrm{~kg} \mathrm{ha}^{-1}$ NPK 15:15:15, F3: $75 \mathrm{~kg} \mathrm{ha}^{-1}$ NPK 15:15:15 + $5 \mathrm{t} \mathrm{ha}^{-1}$ poultry manure.

Table 3. Effect of cutting height and fertilizer application on herbage yield of Amaranthus cruentus L.

\begin{tabular}{|c|c|c|c|c|c|c|}
\hline Treatment & \multicolumn{2}{|c|}{$2017 / 2018$ Cropping season } & \multicolumn{2}{|c|}{$2018 / 2019$ Cropping season } \\
\hline $\begin{array}{c}\text { Fresh herbage } \\
\text { wt }(\mathrm{g} / \mathrm{plt})\end{array}$ & $\begin{array}{c}\text { Fresh herbage } \\
\text { yield } \\
\left(\mathrm{t} \mathrm{ha}^{-1}\right)\end{array}$ & $\begin{array}{c}\text { Dry } \\
\text { herbage yield } \\
\left.(\mathrm{t} \mathrm{ha})^{-1}\right)\end{array}$ & $\begin{array}{c}\text { Fresh herbage } \\
\text { wt } \\
(\mathrm{g} / \mathrm{plt})\end{array}$ & $\begin{array}{c}\text { Fresh } \\
\text { herbage } \\
\text { yield } \\
\left(\mathrm{t} \mathrm{ha}^{-1}\right)\end{array}$ & $\begin{array}{c}\text { Dry } \\
\text { herbage yield } \\
\left(\mathrm{t} \mathrm{ha}^{-1}\right)\end{array}$ \\
\hline $\begin{array}{c}\text { Cutting } \\
\text { height }\end{array}$ & & & & & & \\
\hline C 0 & $15.35 \mathrm{~b}$ & $35.60 \mathrm{~b}$ & $11.50 \mathrm{c}$ & $15.39 \mathrm{~b}$ & $37.00 \mathrm{~b}$ & $13.44 \mathrm{~d}$ \\
\hline C 1 & $16.84 \mathrm{~b}$ & $34.80 \mathrm{~b}$ & $14.20 \mathrm{~b}$ & $32.54 \mathrm{a}$ & $67.10 \mathrm{a}$ & $29.31 \mathrm{c}$ \\
\hline C 2 & $1677 \mathrm{~b}$ & $35.00 \mathrm{~b}$ & $14.10 \mathrm{~b}$ & $26.12 \mathrm{a}$ & $57.41 \mathrm{a}$ & $25.53 \mathrm{~b}$ \\
\hline
\end{tabular}


Falodun EJ and Edafe VE (2020). Not Sci Biol 12(3):719-728

\begin{tabular}{|c|c|c|c|c|c|c|}
\hline C 3 & $2042 \mathrm{a}$ & $49.10 \mathrm{a}$ & $18.20 \mathrm{a}$ & $20.27 \mathrm{a}$ & $48.40 \mathrm{a}$ & $28.32 \mathrm{a}$ \\
\hline Significance & ${ }^{*}$ & ${ }^{*}$ & ${ }^{*}$ & ${ }^{*}$ & ${ }^{*}$ & ${ }^{*}$ \\
\hline Lsd $(0.05)$ & 2.13 & 10.52 & 3.50 & 10.32 & 3.14 & 2.19 \\
\hline $\begin{array}{c}\text { Fertilizer } \\
\text { rates }\end{array}$ & & & & & & \\
\hline F 0 & $18.92 \mathrm{~b}$ & $28.50 \mathrm{c}$ & $9.21 \mathrm{~b}$ & $28.99 \mathrm{a}$ & $47.42 \mathrm{~b}$ & $18.92 \mathrm{c}$ \\
\hline F 1 & $24.88 \mathrm{a}$ & $35.90 \mathrm{bc}$ & $11.59 \mathrm{~b}$ & $37.34 \mathrm{a}$ & $56.22 \mathrm{a}$ & $2241 \mathrm{~b}$ \\
\hline F 2 & $24.12 \mathrm{a}$ & $49.50 \mathrm{a}$ & $15.99 \mathrm{a}$ & $36.10 \mathrm{a}$ & $53.45 \mathrm{a}$ & $21.20 \mathrm{~b}$ \\
\hline F 3 & $25.16 \mathrm{a}$ & $40.50 \mathrm{ab}$ & $13.08 \mathrm{a}$ & $36.25 \mathrm{a}$ & $54.82 \mathrm{a}$ & $25.15 \mathrm{a}$ \\
\hline Significance & $*$ & ${ }^{*}$ & ${ }^{*}$ & $\mathrm{~ns}$ & ${ }^{*}$ & $*$ \\
\hline Lsd $(0.05)$ & 2.13 & 10.52 & 3.50 & 10.32 & 3.14 & 2.19 \\
\hline Interaction & & & & & & $\mathrm{n}$ \\
\hline C*F & $\mathrm{ns}$ & $\mathrm{Ns}$ & $\mathrm{ns}$ & $\mathrm{ns}$ & $\mathrm{ns}$ \\
\hline
\end{tabular}

Means followed by the same alphabets along rows are not significantly different at $5 \%$ level of probability C0: No cutting, C1: $(10 \mathrm{~cm})$, C2: $(15 \mathrm{~cm})$, C3: $(20 \mathrm{~cm})$ above soil level.

F0: Control, F1: $10 \mathrm{t} \mathrm{ha}^{-1}$ poultry manure, F2: $150 \mathrm{~kg} \mathrm{ha}^{-1}$ NPK 15:15:15, F3: $75 \mathrm{~kg} \mathrm{ha}^{-1}$ NPK 15:15:15 + $5 \mathrm{t} \mathrm{ha}^{-1}$ poultry manure.

Table 4. Effect of cutting heights and fertilizer applications on nutrient concentration of Amaranthus cruentus $\mathrm{L}$.

\begin{tabular}{|c|c|c|c|c|c|c|}
\hline \multicolumn{7}{|c|}{ Nutrient concentration $\left(\mathrm{g} \mathrm{kg}^{-1}\right)$} \\
\hline Treatment & $\mathrm{N}$ & $\mathrm{P}$ & $\mathrm{K}$ & $\mathrm{Mg}$ & $\mathrm{Ca}$ & $\mathrm{Na}$ \\
\hline \multicolumn{7}{|c|}{ Cutting height } \\
\hline $\mathrm{CO}$ & $0.01 b$ & $0.42 \mathrm{a}$ & $0.27 \mathrm{~b}$ & $0.37 b$ & $2.10 \mathrm{~b}$ & $0.01 \mathrm{a}$ \\
\hline $\mathrm{C} 1$ & $0.01 \mathrm{~b}$ & $0.41 \mathrm{a}$ & $0.25 b$ & $0.32 \mathrm{~b}$ & $1.94 \mathrm{~b}$ & $0.01 \mathrm{a}$ \\
\hline $\mathrm{C} 2$ & $0.01 \mathrm{~b}$ & $0.48 \mathrm{a}$ & $0.31 b$ & $0.46 b$ & $2.47 \mathrm{~b}$ & $0.20 \mathrm{a}$ \\
\hline $\mathrm{C} 3$ & $0.03 a$ & $8.39 \mathrm{a}$ & $0.44 a$ & $0.63 a$ & $3.32 \mathrm{a}$ & $0.01 \mathrm{a}$ \\
\hline Significance & ${ }^{*}$ & ns & ${ }^{*}$ & ${ }^{*}$ & ${ }^{*}$ & ns \\
\hline LSD $_{0.05}$ & 0.01 & 0.07 & 0.09 & 0.15 & 0.73 & 0.29 \\
\hline \multicolumn{7}{|c|}{ Fertilizer rate } \\
\hline F0 & $0.01 \mathrm{~b}$ & $0.36 \mathrm{a}$ & $0.20 \mathrm{~b}$ & $0.37 \mathrm{a}$ & $1.09 \mathrm{~b}$ & $0.01 \mathrm{a}$ \\
\hline F1 & $0.01 \mathrm{~b}$ & $0.51 \mathrm{a}$ & $0.33 a$ & $0.48 \mathrm{a}$ & $2.76 a$ & $0.01 \mathrm{a}$ \\
\hline $\mathrm{F} 2$ & $0.03 \mathrm{a}$ & $8.32 \mathrm{a}$ & $0.36 \mathrm{a}$ & $0.52 \mathrm{a}$ & $2.66 a$ & $0.21 \mathrm{a}$ \\
\hline F3 & $0.02 \mathrm{ab}$ & $0.51 \mathrm{a}$ & $0.33 \mathrm{ab}$ & $0.41 \mathrm{a}$ & $2.51 \mathrm{a}$ & $0.01 \mathrm{a}$ \\
\hline Significance & $*$ & $\mathrm{~ns}$ & $*$ & $\mathrm{~ns}$ & $*$ & ns \\
\hline LSD $_{0.05}$ & 0.01 & 0.07 & 0.09 & 0.15 & 0.73 & 0.29 \\
\hline
\end{tabular}

Means followed by the same alphabets along rows are not significantly different at $5 \%$ level of probability C0: No cutting, C1: $(10 \mathrm{~cm})$, C2: $(15 \mathrm{~cm})$, C3: $(20 \mathrm{~cm})$ above soil level.

F0: Control, F1: $10 \mathrm{t} \mathrm{ha}^{-1}$ poultry manure, F2: $150 \mathrm{~kg} \mathrm{ha}^{-1}$ NPK 15:15:15, F3: $75 \mathrm{~kg} \mathrm{ha}^{-1}$ NPK 15:15:15 + $5 \mathrm{t} \mathrm{ha}^{-1}$ poultry manure. 
Falodun EJ and Edafe VE (2020). Not Sci Biol 12(3):719-728

Table 5. Effect of cutting heights and fertilizer applications of nutrient uptake of Amaranthus cruentus L.

\begin{tabular}{|c|c|c|c|c|c|c|}
\hline & \multicolumn{6}{|c|}{ Nutrient uptake $\left(\mathrm{kg} \mathrm{ha}^{-1}\right)$} \\
\hline Treatments & $\mathrm{N}$ & $\mathrm{P}$ & $\mathrm{K}$ & $\mathrm{Mg}$ & $\mathrm{Ca}$ & $\mathrm{Na}$ \\
\hline \multicolumn{7}{|c|}{ Cutting height } \\
\hline C0 & $0.68 \mathrm{~d}$ & $29.58 c$ & $14.30 \mathrm{~d}$ & $19.85 c$ & $152.25 \mathrm{~d}$ & $0.06 \mathrm{~d}$ \\
\hline $\mathrm{C} 1$ & $0.85 \mathrm{c}$ & $33.90 \mathrm{~b}$ & $19.00 \mathrm{c}$ & $24.84 \mathrm{~b}$ & $156.33 c$ & $0.14 \mathrm{c}$ \\
\hline $\mathrm{C} 2$ & $1.17 \mathrm{~b}$ & $33.65 b$ & $21.89 \mathrm{~b}$ & $34.91 \mathrm{a}$ & $172.75 b$ & $0.19 \mathrm{~b}$ \\
\hline C3 & $1.67 \mathrm{a}$ & $41.01 \mathrm{a}$ & $24.08 \mathrm{a}$ & $34.45 a$ & $180.58 \mathrm{a}$ & $0.35 \mathrm{a}$ \\
\hline Significance & * & * & $*$ & $*$ & * & * \\
\hline LSD $_{0.05}$ & 0.08 & 1.57 & 1.15 & 1.82 & 3.86 & 0.02 \\
\hline \multicolumn{7}{|c|}{ Fertilizer rate } \\
\hline F0 & $0.93 c$ & $32.69 b$ & $18.35 \mathrm{c}$ & $26.80 \mathrm{~b}$ & $160.16 b$ & $0.13 c$ \\
\hline F1 & $1.07 \mathrm{~b}$ & $34.44 a$ & $19.10 \mathrm{bc}$ & $28.29 \mathrm{ab}$ & $166.33 a$ & $0.19 \mathrm{~b}$ \\
\hline F2 & $1.19 \mathrm{a}$ & $35.30 \mathrm{a}$ & $20.16 \mathrm{~b}$ & $29.08 \mathrm{a}$ & $168.58 \mathrm{a}$ & $0.20 \mathrm{ab}$ \\
\hline F3 & $1.18 \mathrm{a}$ & $35.73 a$ & $21.65 a$ & $29.89 a$ & $166.83 a$ & $0.22 \mathrm{a}$ \\
\hline Significance & ${ }^{*}$ & $*$ & $*$ & $*$ & ${ }^{*}$ & ${ }^{*}$ \\
\hline LSD $_{0.05}$ & 0.08 & 1.57 & 1.15 & 1.82 & 3.86 & 0.22 \\
\hline
\end{tabular}

Means followed by the same alphabets along rows are not significantly different at $5 \%$ level of probability

C0: No cutting, C1: $(10 \mathrm{~cm}), \mathrm{C} 2:(15 \mathrm{~cm}), \mathrm{C} 3:(20 \mathrm{~cm})$ above soil level.

F0: Control, F1: $10 \mathrm{t} \mathrm{ha}^{-1}$ poultry manure, F2: $150 \mathrm{~kg} \mathrm{ha}^{-1}$ NPK 15:15:15, F3: $75 \mathrm{~kg} \mathrm{ha}^{-1}$ NPK 15:15:15 + $5 \mathrm{t} \mathrm{ha}^{-1}$

poultry manure.

Table 6. Effect of cutting heights and fertilizer application on moisture, ash, and crude protein contents Amaranthus cruentus L.

\begin{tabular}{|c|c|c|c|}
\hline Treatments & $\begin{array}{c}\text { Moisture } \\
\text { (\% dry matter content) }\end{array}$ & $\begin{array}{c}\text { Ash content } \\
(\%)\end{array}$ & Crude protein content (\%) \\
\hline \multicolumn{5}{|c|}{ Cutting height } \\
\hline C0 & $3.25 \mathrm{~b}$ & $4.30 \mathrm{~d}$ & $1.09 \mathrm{~d}$ \\
\hline C1 & $2.25 \mathrm{c}$ & $4.82 \mathrm{c}$ & $4.28 \mathrm{c}$ \\
\hline C2 & $3.50 \mathrm{a}$ & $5.05 \mathrm{~b}$ & $5.03 \mathrm{a}$ \\
\hline C3 & $2.00 \mathrm{~d}$ & $5.70 \mathrm{a}$ & $*$ \\
\hline Significance & $*$ & $*$ & $1.84 \mathrm{~d}$ \\
\hline \multicolumn{5}{|c|}{ Fertilizer rate } \\
\hline F0 & $3.00 \mathrm{a}$ & $4.12 \mathrm{~d}$ & $3.06 \mathrm{c}$ \\
\hline F1 & $3.00 \mathrm{a}$ & $5.07 \mathrm{c}$ & $3.60 \mathrm{~d}$ \\
\hline F2F & $2.50 \mathrm{~b}$ & $5.40 \mathrm{a}$ & $4.26 \mathrm{a}$ \\
\hline F3 & $2.50 \mathrm{~b}$ & $5.27 \mathrm{~b}$ & $*$ \\
\hline Significance & $*$ & $*$ & \\
\hline
\end{tabular}

Means followed by the same alphabets along rows are not significantly different at $5 \%$ level of probability

C0: No cutting, C1: $(10 \mathrm{~cm}), \mathrm{C} 2:(15 \mathrm{~cm}), \mathrm{C} 3:(20 \mathrm{~cm})$ above soil level.

F0: Control, F1: $10 \mathrm{t} \mathrm{ha}^{-1}$ poultry manure, F2: $150 \mathrm{~kg} \mathrm{ha}^{-1}$ NPK 15:15:15, F3: $75 \mathrm{~kg} \mathrm{ha}^{-1}$ NPK 15:15:15 + $5 \mathrm{t} \mathrm{ha}^{-1}$ poultry manure.

Table 7. Effect of cutting heights and fertilizer application on fat, crude fibre and carbohydrate content of Amaranthus cruentus $\mathrm{L}$.

\begin{tabular}{|c|c|c|c|}
\hline Treatments & Crude fat content $(\%)$ & Crude fiber content $(\%)$ & Carbohydrate content (\%) \\
\hline \multicolumn{4}{|c|}{$\begin{array}{c}\text { Cutting height } \\
\end{array}$} \\
\hline $\mathrm{CO}$ & $4.72 \mathrm{~d}$ & $2.80 \mathrm{~d}$ & $83.93 a$ \\
\hline $\mathrm{C} 1$ & $5.50 \mathrm{c}$ & $3.80 \mathrm{c}$ & $81.08 \mathrm{~b}$ \\
\hline $\mathrm{C} 2$ & $6.52 b$ & $3.97 \mathrm{~b}$ & $75.25 \mathrm{c}$ \\
\hline $\mathrm{C} 3$ & $7.92 \mathrm{a}$ & $6.22 \mathrm{a}$ & $75.03 \mathrm{~d}$ \\
\hline Significance & ${ }^{*}$ & $*$ & $*$ \\
\hline
\end{tabular}


Falodun EJ and Edafe VE (2020). Not Sci Biol 12(3):719-728

\begin{tabular}{|c|c|c|c|}
\hline \multicolumn{3}{|c|}{ Fertilizers rate } \\
\hline F0 & $5.70 \mathrm{~d}$ & $3.85 \mathrm{c}$ & $81.46 \mathrm{a}$ \\
\hline F1 & $5.95 \mathrm{c}$ & $4.52 \mathrm{~b}$ & $77.84 \mathrm{~b}$ \\
\hline F2 & $6.32 \mathrm{~b}$ & $3.80 \mathrm{~d}$ & $77.06 \mathrm{c}$ \\
\hline F3 & $6.70 \mathrm{a}$ & $4.62 \mathrm{a}$ & $77.01 \mathrm{~d}$ \\
\hline Significance & $*$ & ${ }^{*}$ & ${ }^{*}$ \\
\hline
\end{tabular}

Means followed by the same alphabets along rows are not significantly different at $5 \%$ level of probability

C0: No cutting, C1: $(10 \mathrm{~cm})$, C2: $(15 \mathrm{~cm})$, C3: $(20 \mathrm{~cm})$ above soil level.

F0: Control, F1: $10 \mathrm{t} \mathrm{ha}^{-1}$ poultry manure, F2: $150 \mathrm{~kg} \mathrm{ha}^{-1}$ NPK 15:15:15, F3: $75 \mathrm{~kg} \mathrm{ha}^{-1}$ NPK 15:15:15 + $5 \mathrm{t} \mathrm{ha}^{-1}$

poultry manure.

\section{Discussion}

The soil used for this experiment was below the critical concentration level $\mathrm{N}(0.15 \%), \mathrm{P}(8-10 \mathrm{mg}$ $\left.\mathrm{kg}^{-1}\right), \mathrm{K}\left(0.20 \mathrm{cmol} \mathrm{kg}^{-1}\right), \mathrm{Ca}(2.00 \mathrm{cmol} \mathrm{kg}-1), \mathrm{Mg}\left(0.26 \mathrm{cmol} \mathrm{kg}^{-1}\right)$ needed for arable crop production Agboola and Ayodele (1985). Hence the need for additional soil nutrients to increase the soil fertility. The increase in the vegetative characters especially in the number of leaves and branches as a result of cutting heights compared with the non-cut could probably be due to the fact that cut plants produced more leaves and buds on the stem consequently resulting in a high level of auxin for re-growth. Olufolaji and Tayo (1989) showed that the reaping and regrowth system of vegetable production was superior to a once only total harvest for Amaranthus (Amaranthus cruentus L.). They showed that the total numbers of leaves and branches of Amaranthus were greater, gaining a higher total fresh weight yield, and the total dry weight of various plant parts.

Stem cuttings at several intervals above the ground permit the lateral shoots to grow so that harvesting can be done a greater number of times. This work agrees with the findings of Akanbi et.al., (2009) that cutting height has significant effect on the growth of amaranth. The significant lower number of leaves, stem diameter and yield attributes observed with the non-fertilized plants support the findings of Ojo and Olufolaji (1987) who reported that soils that are inherently low in essential nutrients, reduce the uptake and other physiological processes that cumulate into biomass production. This might be the reasons for observed low dry matter and shoot yield production for plants that were not fertilized. The significant increase in both fresh and dry herbage yield of $A$. cruentus with plants cut at $20 \mathrm{~cm}$ above ground level might be due to accumulation of higher nutrients and presence of more functional nodes from which side shoot re - growth occured. Hence better dry matter and shoot yield production of this cutting height over other cutting heights. These findings corroborate the work of Akanbi (2009) and Robert and Andrew (1989) who stated that repeated cutting of shoot produced higher shoot yield than harvesting once. Maruo et al. (2003) and Takagaki et al. (2003) reported that reaping and regrowth system is feasible for vegetable production especially leafy and shoot type vegetable. The increase in fresh and dry herbage yield observed with F2, (150 $\mathrm{kg} \mathrm{ha}^{-1}$ NPK15:15:15) and F3, (75 $\mathrm{kg} \mathrm{ha}^{-1}$ NPK 15:15:15 $+5 \mathrm{tha}^{-1}$ poultry manure) could be attributed to its higher nutrient concentrations $(\mathrm{N}, \mathrm{P}, \mathrm{K}, \mathrm{Ca}, \mathrm{Mg}$ and $\mathrm{Na}$ ), which increased the availability of nutrients in the soil, leading to increased uptake by $A$. cruentus plants. Thus, enhanced nutrition and increased number of functional nodes which had positive effects on the dry matter production and shoot yield). The higher concentration of $\mathrm{N}, \mathrm{P}, \mathrm{K}$ and $\mathrm{Mg}$ in C3: (plants cut at $20 \mathrm{~cm}$ above ground level) might be due to the fact that the plants absorbed more nutrient from the soil and the absorbed nutrients might have translocated to the new leaves that were formed. Ojeniyi and Odedina (2014) classified NPK and Mg as mobile plant nutrients while Fe, Mn and Ca were classified as immobile nutrients. Nutrient uptake for fertilizer rates revealed that plants that received fertilizer had more nutrient concentration than nonfertilized plants. This corroborates the findings of Ademola et al. (2019), who stated the significant increase in nutrient concentration as influenced by application of different fertilizer materials. The proximate composition values obtained in this study are slightly higher than those of Karuki et al. (2013) except for the crude protein. The increase in ash, crude protein, fat and crude fibre content of the shoot with increase in 
cutting height (especially in $\mathrm{C} 3$ ) and at $\mathrm{F} 3$ is an evident of an increase in the nutritional quality of $A$ cruentus. This might be as a result of migration of nutrients to the newly formed shoots that were harvested.

\section{Conclusions}

This research work showed that cutting heights enhanced the growth, fresh herbage and dry herbage yield of $A$. cruentus. Sole application (F1) of $150 \mathrm{~kg} \mathrm{ha}^{-1} \mathrm{NPK}$ 15:15:15 produced the highest ash content while the non - cut plants produced the highest carbohydrate content. Plants cut at $20 \mathrm{~cm}$ above soil level produced the highest cumulative yield, nutrient uptake, fat content, crude fibre content and protein content of $A$. cruentus. These positive effects were more pronounced when the plants were cut at $\mathrm{C} 3$ ( $20 \mathrm{~cm}$ above soil level) and cropped with (F3). Cropping Amaranth with a combined application of fertilizer at $75 \mathrm{~kg} \mathrm{ha}^{-1} \mathrm{NPK}$ 15:15:15 $+5 \mathrm{tha}^{-1}$ poultry manure and subsequent harvesting cut at $20 \mathrm{~cm}$ above soil level are thereby suggested for Amaranth farmers.

\section{Authors' Contributions}

This work was carried out in collaboration between the authors. EJF designed the study, wrote the protocol, and wrote the first draft of the manuscript. EVE managed the literature search and experimental process. Both authors read and approved the final manuscript.

\section{Acknowledgements}

Authors are grateful to the University of Benin, Nigeria for the enabling environment to carry out this research and to DrS.A. Ogedegbe for data analysis.

\section{Conflict of Interests}

The authors declare that there are no conflicts of interest related to this article.

\section{References}

Ademola JA, Aderemi FT, Olunloyo AA, Olomola DB, Elesho RO (2019). Evaluation of different fertilizer types for improved growth and yield of grain amaranth (Amaranthus cruentus L.). International Journal of Advanced Academic Research Sciences, Technology and Engineering 5(10):26-34.

Adeoluwa OO, Akinyemi O (2014). Amaranths (Amaranthus viridis) dry matter and soil qualities: organic vs inorganic fertilizers. In: Rahmann G, Aksoy U (Eds). Proceedings of the 4th ISOFAR Scientific Conference, Building Organic Bridges, at the Organic World Congress 2014, 13-15 October, Istanbul, Turkey pp 879-882.

Adeoye GO, Sridhar MKC, Adeoluwa OO, Oyekunle M, Makinde EA, Olowoake AA (2008). Comparative evaluation of organo-mineral fertilizer (OMF) and mineral fertilizer (NPK) on yield and quality of maize (Zea mays L.). Nigerian Journal of Soil Science 18:132-137.

Agboola AA, Ayodele OJ (1985). Prospects and problems of using soil testing for adoption of fertilizer use in Ekiti-Akoko Agricultural development area. Proceedings of the workshop on appropriate technologies for farmers in semi-arid West Africa, April 2-5, 1985, Purdue University West Lafayette, pp 123-126. 
Akanbi WB, Adeyeye AS, Ogunrinde JO, Babajide PA, Ajibola AT, Ilupeju EAO, Akinfasoye JA (2009). Effect of organic fertilizer and cutting height on growth, shoot yield and nutrient uptake of amaranth (Amarantus cruentus). Acta Satech 3(1):7-14.

Akanbi WB, Togun AO (2002). Productivity and Influence of maize stover compost on growth, yield and nutrient uptake of amaranth. Scientia Horticulture 93(1):1-8. https://doi.org/10.1016/S0304-4238(01)00305-3

Akanbi WB, Togun AO, Adediran JA, Ilupeju EAO (2010). Growth, dry matter and fruit yields components of okra under organic and inorganic sources of nutrients. American-Eurasian Journal of Sustainable Agriculture 4(1):113.

AOAC (1990). Official methods of analysis. 17th Edition Association of Official Analytical Chemist. Washington DC, USA.

Falodun EJ, Ehigiator JO, Egharevba RKA (2013). Growth and yield of onion as influenced by organic and inorganic fertilizer in Edo Rainforest of Nigeria. Agro-Science 12(3):15-24. https://doi.org/10.4314/as.v12i3.3

Gundlade MO, Adeyemi EA, Gunleti DOO, Ibiyomi PS (2011). Effect of cocoa pod husk, urea fortified cocoa pod husk and NPK fertilizers on the growth and yield of Solanum macrocarpon cultivation. International Journal of Organic Agriculture Research and Development 3:1-8.

ISTA (1993). International rules for seed testing association. Supplementary rules. Seed Science and Technology.

Kariuki S, Sila D, Kenji G (2013). Nutritional profile of amaranth grain varieties grown in Kenya. Journal of Agriculture and Food Technology 17:19-25.

Law-Ogbomo KE, Remison SU, Jombo EO (2012). Effect of organic and inorganic fertilizer on the productivity of Amaranthus cruentus in an ultisol environment. Magnesium 1:1-44. https://doi.org/10.5897/IJPPB11.028

Maruo T, Akimoto S, Wada N, Ito M, Takagaki M, Shinohara Y (2002). New leaf vegetable production system using automatic reaping harvester and rail system. In: XXVI International Horticultural Congress: Asian Plants with Unique Horticultural Potential: Genetic Resources, Cultural 620:63-70.

Mnzava NA, Masam AM (1985). Regeneration potential, leaf and seed yield of vegetable amaranth (Amaranthus cruentus L.), as a function of initial topping heights. In: IX African Symposium on Horticultural Crops 153:151-160. https://doi.org/10.17660/ActaHortic.1985.153.20

Moyin-Jesu EI (2002). Use of plant residues for improving soil fertility, pod nutrients, root growth and pod weight of okra $\begin{array}{llll}\text { (Abelmoschus esculentus } & \text { L.). } & \text { Bioresource } & \text { Technology 98(11):2057-2064. }\end{array}$ https://doi.org/10.1016/j.biortech.2006.03.007

NIHORT (1986). National Horticulture Research Institute. Advances in fruit and vegetable research at NIHORT, Ibadan, Nigeria.

Ojeniyi SO, Odedina AS (2014). Basic plant nutrients. Dominion publishers, Ibadan, Nigeria, pp 60.

Ojo OD, Olufolaji AO (1987). Optimum NPK fertilizer rates for growth and yield of Solanum macrocrapon (cv. Igbagba). Journal of Vegetable Crop Production 3(1):73-77. https://doi.org/10.1300/J068v03n01_09

Olufolaji AO, Tayo AO (1989). Performance of four morphotypes of Amaranthus cruentus L. under two harvesting methods. Tropical Agriculture 66(3):273-276.

Robert KM, Andrew JW (1989). An introduction to the physiology of crop yields. Pub. US John Wiley and Sons Inc. New York.

Takagaki T, Amuka S, Maruo T, Sukprakan S, Shinohara Y (2003). Application of reaping method for harvesting leafy vegetables grown in capillary hydroponic system. In: XXVI International Horticultural Congress: Asian Plants with Unique Horticultural Potential: Genetic Resources, Cultural 620:71-76.

Tindall HD (1986). Vegetables in the tropics. Macmillan, 36-41 Education LTD, Hampshire, UK pp 36-41.
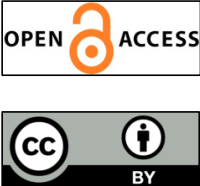

The journal offers free, immediate, and unrestricted access to peer-reviewed research and scholarly work. Users are allowed to read, download, copy, distribute, print, search, or link to the full texts of the articles, or use them for any other lawful purpose, without asking prior permission from the publisher or the author.

License - Articles published in Notulae Scientia Biologicae are Open-Access, distributed under the terms and conditions of the Creative Commons Attribution (CC BY 4.0) License.

(c) Articles by the authors; SHST, Cluj-Napoca, Romania. The journal allows the author(s) to hold the copyright/to retain publishing rights without restriction. 